\title{
A methodological approach for the optimisation of modern rotary blood pumps
}

\author{
D. Palmieri ${ }^{1}$, G. Vezzani ${ }^{1}$, F. Lagasco ${ }^{1}$, P. Nüsser ${ }^{2}$, K. Graichen ${ }^{2}$ \\ \& B. Wieneke ${ }^{3}$ \\ ${ }^{I} D^{\prime}$ Appolonia S.p.A., Italy \\ ${ }^{2}$ Berlin Heart AG, Germany \\ ${ }^{3}$ LaVision GmbH, Germany
}

\begin{abstract}
Particle Image Velocimetry (PIV) analysis and Computational Fluid Dynamics (CFD) simulations have been performed on an innovative prototype of an implantable rotary axial pump. Numerically and experimentally estimated velocity and shear stress fields have been compared on several planes at different distances from the pump axis. An excellent agreement was generally observed in terms of mean flow velocity and viscous stresses, while the congruence of turbulent stresses was very good in some cases and less accurate in others. Further effort will be needed on both the numerical and the experimental side to better characterise near-wall flow features.
\end{abstract}

Keywords: blood pump, PIV, CFD.

\section{Introduction}

Mechanical support offered by rotary pumps is well known in cardiac diseases therapy. The range of applications spans from cardiac support prior to transplantation (Bridge to Transplant) to heart assistance to recovery (Bridge to Recovery). Rotary blood pumps are attractive in this respect because of its simplicity and small size. In particular, implantable pumps must be extremely compact, and overcome the problems of inaccessibility by highly reliable shaft seals and bearings. Technical evolution of these devices offers great perspectives for the continuous increase of the patient's mobility and comfort.

The hydrodynamic design of such devices is very complex because of the detailed characterisation of the blood flow required to identify potential areas of 
blood damage and stasis induced by the flow field within the device. The verification and optimisation process is experimentally carried out by means of flow visualisation technique such as PIV, and haemolysis tests. The opportunity of developing accurate CFD models can offer great advantages in reducing development time and costs and in providing complementary support to device certification.

To date, the majority of blood pump investigations involving CFD have concerned centrifugal pumps [1],[2],[3],[4],[5],[6], while a more limited amount of work has been published on axial pumps [7],[8],[9]. Validation of numerical models has been performed by comparison with experimental results of pressureflow characteristics [5],[7],[9], flow field visualisation [1],[7], and haemolysis levels [9],[10]. Numerical simulations have been used to evaluate the relative performance of different design configurations, for example in terms of overall efficiency [4],[7] or detailed features such as impeller clearance and shape [1],[2],[9] or washout holes [11], to calculate stresses within the gaps between rotating and fixed parts [3],[12], and to evaluate blood damage based on the exposure time to a given stress level [6],[8],[9].

This paper presents the results of a concerted action, developed in the frame of a European Commission funded project (SMART-PIV), of an innovative implantable axial pump prototype manufactured by Berlin Heart AG. The proposed methodology is based on accurate CFD simulations, experimentally validated by means of advanced miniaturised PIV techniques.

\section{Pump prototype description}

The pump prototype is composed by a rotating part (rotor) with a single continuous spiral vane on the side of the flow inlet, and a fixed three vanes outflow guide (stator) on the outlet side, fig. 1.

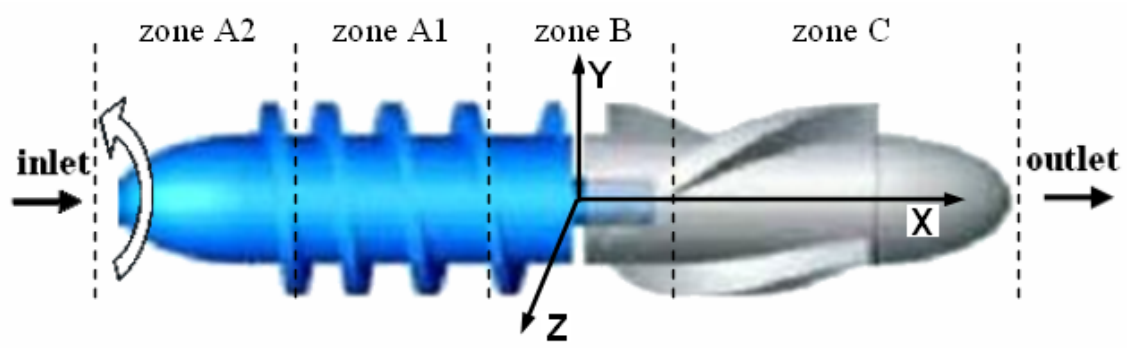

Figure 1: $\quad$ Pump prototype geometry.

The rotor and stator sections, whose length is $24.4 \mathrm{~mm}$ and $20.3 \mathrm{~mm}$, respectively, are enclosed in an external cylindrical housing having an inner diameter of $10 \mathrm{~mm}$. The height of the rotor and stator vanes is $1.5 \mathrm{~mm}$. A nominal gap of $0.05 \mathrm{~mm}$ exists between the outer edge of rotor vanes and the housing. 
The rotor is driven by a motor whose shaft is aligned with the pump axis. The appropriate rotor speed is selected to ensure the prescribed pressure difference and flow rate of the blood flow leaving the pump. Based on preliminary tests, a reference operating rotational speed of $15500 \mathrm{rpm}$ was selected, corresponding to a stable flow rate of $1 \mathrm{l} / \mathrm{min}$.

\section{Experiments}

\subsection{Experimental setup}

Experiments were acquired with a 2D PIV system on a real scale model of the pump, whose external housing is realised in Polymethyl Methacrylate (PMMA) and the rotor and outlet guide in epoxy resin by stereolithography. The fluid circulating in the mock-loop is a special fraction of kerosene, with a refractive index nearly matching that of PMMA. The difference of refractive index between the fluid and the material of inner parts is not such to influence measurement accuracy.

The test item was illuminated by a lightsheet with a thickness of about 150 $\mu \mathrm{m}$, generated by a $532 \mathrm{~mm}$ wavelength double cavity pulsed Nd-Yag laser. The laser light, scattered by $9.5 \mu \mathrm{m}$ PMMA particles suspended in the fluid, was captured by a $1376 \times 1040$ CCD camera with the viewing direction perpendicular to the light sheet, fig. 2.

In order to avoid interfering reflections from the walls of the pump model, that would increase the signal-to-noise ratio especially in the near wall regions, seeding particles were doped with Rhodamin B and a $540 \mathrm{~nm}$ edge wavelength fluorescent filter was placed directly in front of the camera.

Velocity data were obtained by statistic correlation of particles position in couples of images produced by two very close laser shots (double pulse). The duration of each single shot was $6 \mathrm{~ns}$, while two consecutive shots composing a double pulse were separated by a delay variable from 25 to $100 \mu$ s, depending on the flow velocity expected in the particular zone under investigation. The laser was operated with a frequency of $5 \mathrm{~Hz}$ for generation of successive double pulses and an energy of $100 \mathrm{~mJ}$ for a single shot.

The pump domain investigated included the rotor, the stator and the upstream and downstream regions shown in fig. 1. The flow in the inlet and outlet sections was considered to show no significant features with respect to potential blood damage. As the camera field-of-view was insufficient to capture the whole domain of interest, separate measurements were performed in four different zones along the pump axis (zones A1 and A2 for the inlet and rotor region, zone $\mathrm{B}$ for the transition region between rotor and stator, and zone $\mathrm{C}$ for the outlet stator region). Velocity field measurements were acquired for all zones in different planes at 2.5, 3, 3.5, 4 and $4.5 \mathrm{~mm}$ offset from the pump axis (along the $\mathrm{Z}$-axis in fig. 1). The camera and light sheet position was kept fixed, while only the pump model was translated in the directions parallel to its axis and normal to the light sheet in order to acquire the desired volume scanning, as shown in fig. 2. This has the great advantage of minimising the complexity of test equipment and the associated cost, increasing at the same time measurement accuracy. 


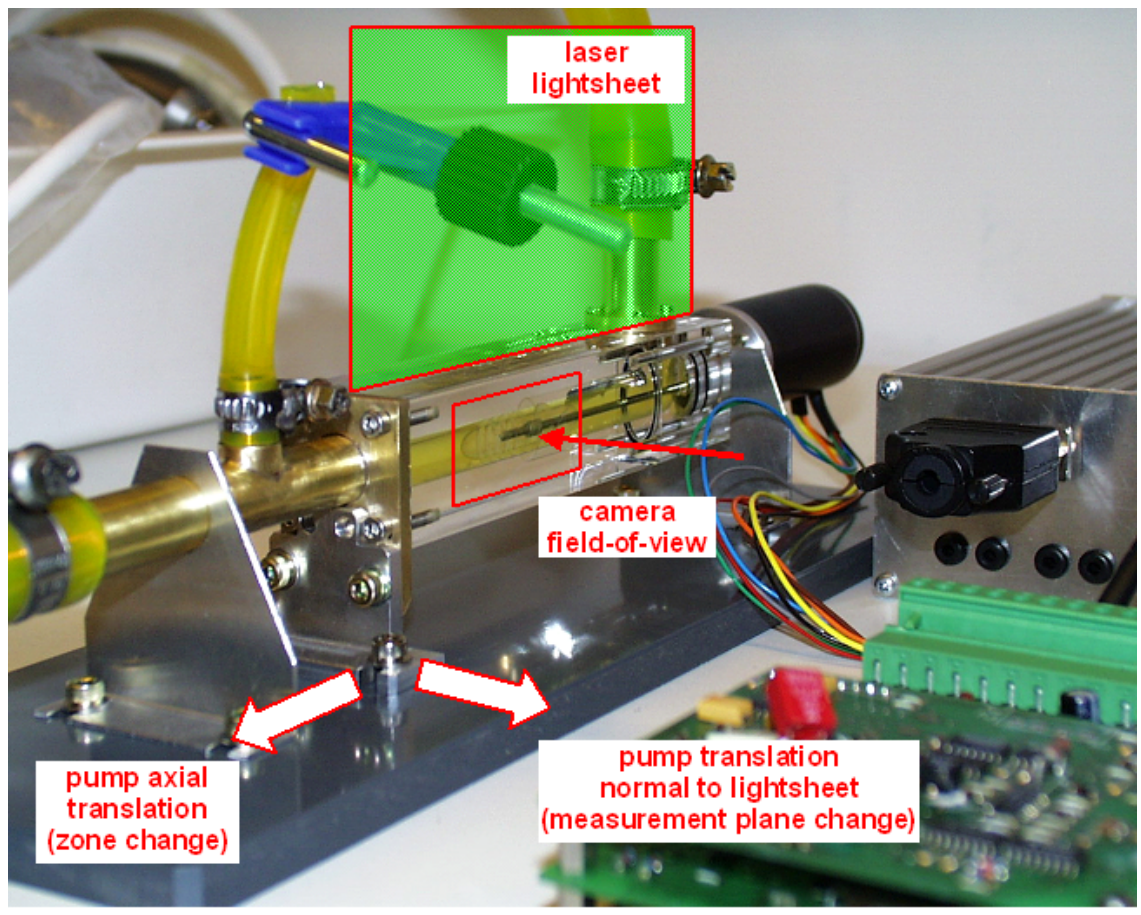

Figure 2: $\quad$ Experimental setup.

In order to resolve the flow mean characteristics in the regions where a pronounced dependency on the rotational angle (phase dependency) is expected, namely within the rotor-stator interaction zone, the pump model was provided with a special "trigger box" generating a TTL pulse per revolution and fed by the signal of one of three hall sensors of the driving motor. The triggering TTL signal is directly fed into a programmable triggering unit connected to the camera, thus allowing the acquisition of the desired phase averaging at a given angular position.

\subsection{Experimental data processing}

Preliminary analysis indicated significant flow dependence on the rotation angle (phase) in zone B, while in zones A1, A2 and C flow features are sufficiently independent on the rotor-stator relative position. Therefore, in zone B images have been recorded during an entire revolution in steps of 10 degrees, and at a single phase in zones A1, A2 and C. 300 PIV images were recorded at each phase and spatial location.

About 40000 images were recorded during the whole experiment sizing to $150 \mathrm{~GB}$, thus requiring a sufficiently automated procedure in order to minimise processing time. An automatic mask was first generated for all measurement points (phases and spatial positions), based on the Root Mean Square (RMS) of 
image intensity at a given pixel over all 300 images. A standard PIV processing has been performed with interrogation window sizes of $64 \times 64$ pixel with $75 \%$ overlap, resulting in effective vector spacing of $0.18 \mathrm{~mm}$. Finally, the 300 vector fields have been analysed to extract, for each phase and spatial position, the mean kinetic and turbulent kinetic energy, the viscous Maximum Shear Strain rate (MSS) based on the eigenvalues of the velocity gradients tensor and therefore independent of the orientation of the coordinate axis, the specific Reynolds stress in-plane component $\overline{u^{\prime} v^{\prime}}$ and the corresponding maximum Turbulent Shear Stress $\mathrm{TSS}_{\max }$ [13], derived from the eigenvalues of the Reynolds shear stress tensor analogously to MSS.

A vector spacing of $0.18 \mathrm{~mm}$ does not allow to measure wall shear stresses close to the walls, being a typical boundary layer thickness around $80 \mu \mathrm{m}$. For this purpose a specific PIV processing scheme was employed, consisting in the use of smaller interrogation windows and summing up all correlation planes before finding the highest correlation peak ("ensemble averaging", [14]). In this way a single average vector field is computed. Data have been processed with an interrogation window size of $16 \times 16$ pixel, corresponding to a vector spacing of $42 \mu \mathrm{m}$.

\section{Numerical simulations}

\subsection{Model description}

Appropriate inlet and outlet sections were included upstream the rotor and downstream the stator, respectively, in order to apply boundary conditions at sufficient distance from the zone of highest interest for flow analysis. The model consists of three separate grids for the inlet, rotor and stator/outlet sections, whose interfaces have the same spatial location but are physically distinct, fig. 3 . Regions within the rotor and stator vanes, as well as inlet and outlet terminal sections, were modelled by a structured hexahedral grid. Spacing between the rotor and stator and their hub tips, possessing less regular geometry, was modelled with unstructured tetrahedral or hexahedral meshes.

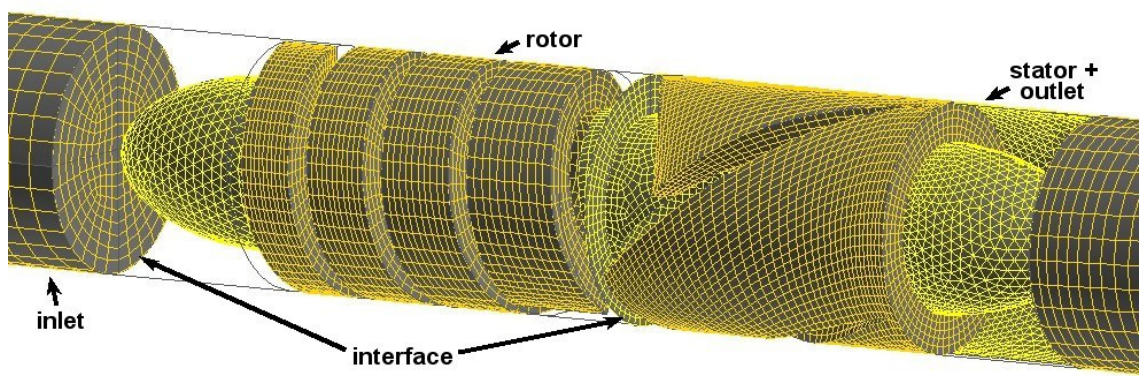

Figure 3: Numerical grid. 
The grid representing the rotor rotates at the prescribed operating speed around the pump axis, and numerical data are interpolated across the interfaces at each time step of the relative motion with respect to the fixed regions. Boundary conditions were set to ensure the operative constant flow rate and a reference zero pressure at the inlet section. No-slip condition was assumed at all wall surfaces. The fluid was considered to be incompressible and Newtonian, with density of $840 \mathrm{~kg} / \mathrm{m}^{3}$ and viscosity of $0.0036 \mathrm{~kg} / \mathrm{m} \cdot \mathrm{s}$.

The narrow gap between the rotor vane and the outer casing was not included in the numerical model to avoid the associated enormous increase of the overall grid size and, consequently, of computation time.

\subsection{Grid dependence}

Sensitivity analyses have been performed with three different mesh resolutions. The smallest cell size in the model was always located in region between the rotor and stator vanes, where the more complex flow pattern and higher gradients are expected. The three analysed meshes were characterised by 5, 10 and 15 uniform size cells within the height of the rotor and stator vanes, respectively. In all cases cell size smoothly increased towards the inlet and outlet regions, where a more uniform flow is envisaged.

Table 1 reports the mean value and standard deviation of the regime total pressure on the outlet section obtained with the three models, representing the overall pressure difference between the pump inlet and outlet and considered as representative variable of the analysis.

Table 1: $\quad$ Grid dependence analysis.

\begin{tabular}{|c|c|c|c|c|c|c|}
\hline case & $\begin{array}{c}\text { nr. of cells } \\
\text { through } \\
\text { vanes } \\
\text { height }\end{array}$ & $\begin{array}{c}\text { cells size } \\
\text { in vanes } \\
\text { height } \\
(\mathrm{mm})\end{array}$ & $\begin{array}{c}\text { total nr. } \\
\text { of cells in } \\
\text { the model }\end{array}$ & $\begin{array}{c}\text { residuals } \\
\text { convergence } \\
\text { threshold }\end{array}$ & $\begin{array}{c}\text { outlet } \\
\text { pressure } \\
\text { mean value } \\
(\mathrm{Pa})\end{array}$ & $\begin{array}{c}\text { outlet } \\
\text { pressure } \\
\text { std. dev. } \\
(\%)\end{array}$ \\
\hline 1 & 5 & 0.3 & 120,400 & $1 \cdot 10^{-4}$ & 19425 & 5.0 \\
\hline 2 & 10 & 0.15 & 318,966 & $1 \cdot 10^{-4}$ & 13760 & 5.1 \\
\hline 3 & 15 & 0.1 & 918,668 & $1 \cdot 10^{-4}$ & 12025 & 6.8 \\
\hline $3 \mathrm{a}$ & 15 & 0.1 & 918,668 & $5 \cdot 10^{-6}$ & 12086 & 3.3 \\
\hline
\end{tabular}

All three models were initially ran with a convergence threshold of equation residuals of $10^{-4}$. After several time steps, in the highest resolution case such threshold was lowered to $5 \cdot 10^{-6}$, in order to evaluate the effect on the oscillations around the mean value observed in the regime solution.

The difference in the predicted outlet pressure progressively reduces with increasing resolutions, indicating a convergence trend to an asymptotic value. Furthermore, the reduction of the residuals threshold reduces the standard deviation around the mean value (case 3a). Refinement achieved in the sensitivity analysis was considered a sufficient counterbalance between grid independence and required computational resources. 


\subsection{Turbulence model}

Turbulent flow simulations were performed with the finest grid by employing the Standard, Renormalization Group (RNG) and Realizable k- $\varepsilon$ models of CFD code (Fluent) libraries. The k- $\varepsilon$ models have been used in the majority of numerical work on heart pumps [1],[2],[4],[5],[6],[9],[11]. Furthermore, they are specifically conceived for the study of flow core turbulence, on which present study is focused. A turbulence intensity of $7 \%$, derived from experimental data, was applied to the boundary of numerical domain. The CFD code standard wall functions were used for near-wall flow calculation.

\section{Comparison of experimental and numerical results}

Numerical and experimental results compared favourably in most cases. In particular, the velocity field and viscous strain closely matched in terms of both maximum values and spatial position. Some light spatial misalignments were observed mainly along the pump axis direction ( $\mathrm{X}$ axis), due either to small geometric differences between the manufactured prototype and the CAD layout imported in the numerical model, or to geometry approximation induced by the automatic mask algorithm in processing experimental images. The agreement of turbulent stresses was very good in some cases and less accurate in others, being observed differences scaled up to $50 \%$ on peak values.

As example, Figures 4, 5 and 6 show the experimental and numerical fields of MSS, in-plane velocity and $\mathrm{TSS}_{\max }$ in the $\mathrm{Z}=3.5 \mathrm{~mm}$ plane at phase angle of $0^{\circ}$ (corresponding to crossing of rotor vane tip with the stator vane on the measurement plane), $120^{\circ}$ and $240^{\circ}$ (corresponding to crossing of rotor vane tip with the two following stator vanes), respectively. A comparison of variable profiles at a given plane section (arrowed line in the pictures) is also reported.

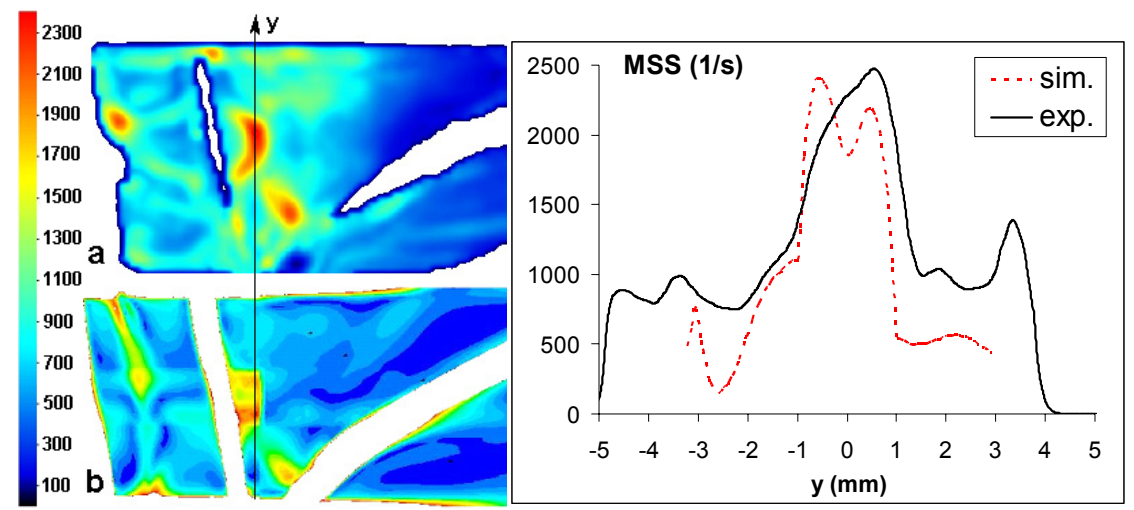

Figure 4: $\quad$ MSS field in zone $B$ at $Z=3.5 \mathrm{~mm}, 0^{\circ}$ phase angle, experimental (a), numerical (b) and profiles comparison at fixed $\mathrm{x}$ position. 
Main peak of MSS, fig. 4, and $\mathrm{TSS}_{\max }$, fig. 6, differ by about $3 \%$ and $7 \%$, respectively, being spatial gradients also in good agreement. Small differences in main peak location and secondary peaks value are observed. Fig. 5 shows the excellent agreement of velocity data in terms of both peak and spatial gradients.

The Standard k- $\varepsilon$ model, through which reported numerical results were obtained, provided the best agreement with tests with respect to the RNG and Realizable k- $\varepsilon$ models.
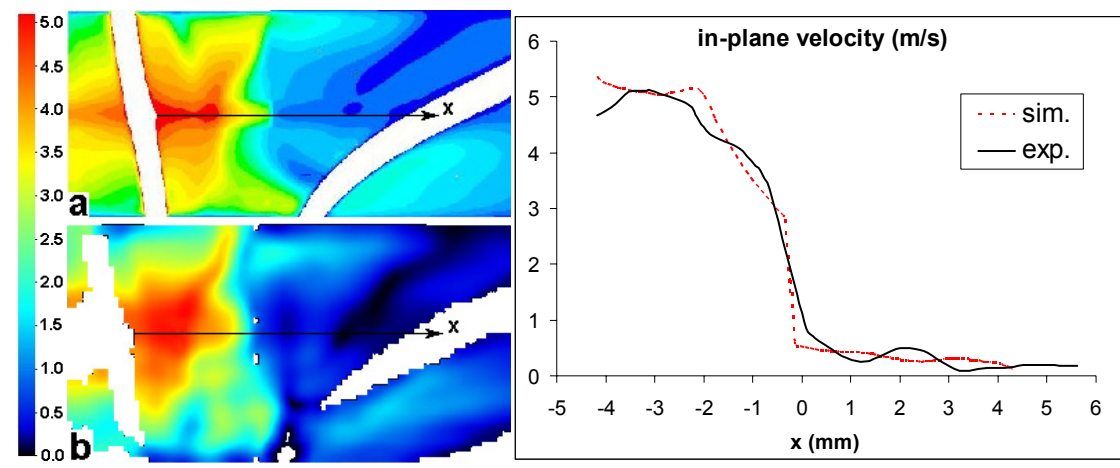

Figure 5: Velocity field in zone $B$ at $Z=3.5 \mathrm{~mm}, 120^{\circ}$ phase angle, experimental (a), numerical (b) and profiles comparison at fixed $y$ position.
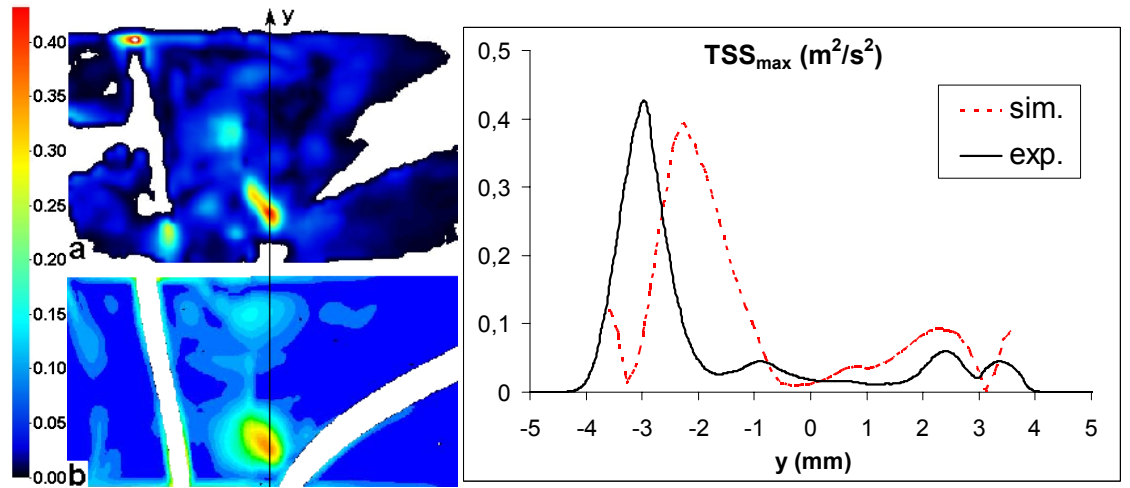

Figure 6: $\mathrm{TSS}_{\max }$ field in zone $\mathrm{B}$ at $\mathrm{Z}=3.5 \mathrm{~mm}, 240^{\circ}$ phase angle, experimental (a), numerical (b) and profiles comparison at fixed $\mathrm{x}$ position.

The better overall agreement between experimental and numerical values of mean flow quantities such as velocity and viscous stresses is justified by their moderate dependence on the number of acquired experimental images, and by their direct calculation as independent variables from the governing equation of numerical models. On the other hand, a larger number of experimental 
acquisitions is required to minimise the deviation of turbulent (time-averaged) shear stresses, while in the used numerical models their calculation is affected by the limitations underlying the Boussinesq's hypothesis:

$$
-\rho \overline{u_{i}^{\prime} u^{\prime}}{ }_{j}=\mu_{t}\left(\frac{\partial u_{i}}{\partial x_{j}}+\frac{\partial u_{j}}{\partial x_{i}}\right) \quad(i \neq j)
$$

In this study, both experimental and numerical data provided maximum viscous stresses in the fluid domain around $10 \mathrm{~Pa}$. Maximum turbulent stresses were found to be generally of the same order of magnitude, but some localized spots with peak values up to about $600 \mathrm{~Pa}$ were experimentally observed in the rotorstator interaction region in the plane at $Z=3.5 \mathrm{~mm}$ from the pump axis, while corresponding numerical peaks resulted to be about $50 \%$ lower. The higher mismatching of numerical and experimental data in this plane can be associated to disturbances induced by its proximity to the wall of pump central body, whose nominal diameter is $3.45 \mathrm{~mm}$. Wall shear strain rate up to $10^{4} \mathrm{~s}^{-1}$ have been preliminarily identified close to the rotor blades and in the gaps using the "ensemble averaging" processing scheme.

Maximum experimental and numerical stresses obtained in this study were lower than Red Blood Cells (RBC) stress damage thresholds of $719 \mathrm{~Pa}$ [2] or 800 $\mathrm{Pa}$ [15] reported in the literature. However, many authors relate haemolysis threshold to a combination of shear stress level and exposure time along blood particles streaklines within the medical device [6],[8],[9],[10].

\section{Conclusions}

A methodology for the design optimisation of rotary blood pumps has been identified and validated by PIV measurements and CFD simulations on an innovative implantable axial pump prototype. Even if further effort is needed to accurately predict flow field and stress levels in the near-wall regions, the appropriate combination of experimental and numerical analysis has revealed to be a very efficient and reliable approach to characterise blood damage within such complex and small devices, thus enhancing confidence in their extensive use and affordability.

\section{References}

[1] Miyazoe, Y. et al, Computational Fluid Dynamic Analyses to Establish Design Process of Centrifugal Blood Pumps. Artificial Organs, 22(5), pp. 381-385, 1998.

[2] Masuzawa, T. et al, Development of Design Methods of a Centrifugal Blood Pump with a Fluid Dynamic Approach: Results in Hemolysis Tests. Artificial Organs, 23(8), pp. 757-761, 1999.

[3] Allaire, P.E., Wood, H.G., Awad, R.S. \& Olsen, D.B., Blood Flow in a Continuous Flow Ventricular Assist Device. Artificial Organs, 23(8), pp. 769-773, 1999. 
[4] Tansley, G., Vidakovic, S. \& Reizes, J., Fluid Dynamic Characteristics of the VentrAssist Rotary Blood Pump. Artificial Organs, 24(6), pp. 483487, 2000.

[5] Watanabe, N. et al, Simulation of the BP-80 Blood Pump. Artificial Organs, 25(9), pp. 733-739, 2001.

[6] Song, X. et al, Quantitative Evaluation of Blood Damage in a Centrifugal VAD by Computational Fluid Dynamics. Journal of Fluids Engineering, 126, pp. 410-418, 2004.

[7] Burgreen, G.W., Antaki, J.F., Wu, Z.J. \& Holmes, A.J., Computational Fluid Dynamics as a Development Tool for Rotary Blood Pumps. Artificial Organs, 25(5), pp. 336-340, 2001.

[8] Apel, J. et al, Assessment of Hemolysis Related Quantities in a Microaxial Blood Pump by Computational Fluid Dynamics. Artificial Organs, 25(5), pp. 341-347, 2001.

[9] Mitoh, A. et al, Computational Fluid Dynamics Analysis of an IntraCardiac Axial Flow Pump. Artificial Organs, 27(1), pp. 34-40, 2003.

[10] Garon, A. \& Farinas, M., Fast Three-dimensional Numerical Hemolysis Approximation. Artificial Organs, 28(11), pp. 1016-1025, 2004.

[11] Tsukamoto, Y. et al., Computational Fluid Dynamics Analysis of a Centrifugal Blood Pump with Washout Holes. Artificial Organs, 24(8), pp. 648-652, 2000.

[12] Qian, Y. \& Bertram, C.D., Computational Fluid Dynamics Analysis of Hydrodynamic Bearings of the VentrAssist Rotary Blood Pump. Artificial Organs, 24(6), pp. 488-491, 2000.

[13] Grigioni, M. et al, On the monodimensional approach to the estimation of the highest Reynolds shear stress in a turbulent flow. J. Biomech, 33(6), pp. 701-708, 2002.

[14] Meinhard, C.D. et al, A PIV Algorithm for Estimating Time-Averaged Velocity Fields. Proc. of Optical Measurements and Image Processing in Fluid Flows, $3^{\text {rd }}$ ASME/FMSE Fluids Engineering Conference, San Francisco, 1999.

[15] Lu, P.C., Lai, H.C. \& Liu, J.S., A reevaluation and discussion on the threshold limit for hemolysis in a turbulent shear flow. J. Biomech., 34(10), pp. 1361-1364, 2001. 